\title{
First-principles electron dynamics simulation study of high intensity laser irradiation on crystal systems: Photon energy dependent energy transfer
}

\author{
Atsushi Yamada ${ }^{1, *}$, and Kazuhiro Yabana ${ }^{1}$ \\ ${ }^{1}$ Center for Computational Sciences, University of Tsukuba, 1-1-1 Tennodai, Tsukuba, Ibaraki 305-8577, Japan
}

\begin{abstract}
Nonlinear interaction between femtosecond laser pulse and simple dielectric solid, silicon, at an intensity range around the damage threshold has been investigated by first-principles calculations based on time-dependent density functional theory (TDDFT). Intensity and frequency dependences are discussed making comparison with the Keldysh theory.
\end{abstract}

\section{Introduction}

Light-matter interaction in an intensity range around the damage threshold has been actively and successfully explored by experimental studies aiming for ultrafast control of electron dynamics and non-thermal laser processing, while theoretical description of this area has not been well developed due to highly nonlinear and complex nature of the interaction. The fundamental energy transfer process in the interaction between the highintensity laser pulse and dielectrics are one photon absorption for a laser frequency above the bandgap and multiphoton absorption and tunnelling ionization for a frequency below the bandgap. A representative theory that describes these mechanisms is Keldysh theory [1]. This has been quite successful to explain various features of the nonlinear electronic excitations, however, approximations and parameters are included in the formula. On the other hand, a recently developed first-principles computational method, time-dependent density functional theory (TDDFT), can be another powerful tool to be able to describe highly nonlinear interactions between high-intensity laser pulses and electrons in various matters with less approximations and without empirical parameters as long as the functional adopted in the calculation does not include parameters for a specific purpose. In this paper, we present an analysis for the energy transfer from a pulsed electric field to electrons in a unit cell of a representative simple crystalline solid, silicon, using TDDFT. Dependence on frequency as well as intensity of the pulsed electric field is shown to understand the mechanisms. Then, we compare the calculated TDDFT results with the Keldysh theory. The comparison will figure out usefulness and limitation of the Keldysh theory.

\section{Theoretical method}

Electron dynamics is described by the time-dependent Kohn-Sham (KS) equation for Bloch orbitals following standard framework of TDDFT with the spatial lattice periodicity [2], where the orbitals are expressed by real space grids. The interaction between a high-

\footnotetext{
*Corresponding author: ayamada@ccs.tsukuba.ac.jp
} 
intensity pulsed light and electrons in dielectrics is expressed by a vector potential $\boldsymbol{A}(t)$ in the KS Hamiltonian using the velocity gauge. We use time profile of cosine-squared envelope shape, $\boldsymbol{A}(t)=-\left(A_{0} / \omega\right) \cos ^{2}(\pi t / T) \cos (\omega t) \boldsymbol{e}_{\mathrm{c}}(0<t<T)$, where $\omega$ is the average frequency and $T$ is the duration. The polarization vector is chosen to be $c$ direction.

For a reliable description to reproduce energetic features quantitatively, we employ the TB-mBJ potential, which is a meta-GGA potential and is known to accurately reproduce bandgap energies of dielectrics. We determine a parameter $c$ in the potential to reproduce the energy of the indirect bandgap of the material. The tuned value is 1.04 for silicon. Calculated dielectric function is in reasonable agreement with experimental data, especially showing that the rising position of the first main peak in the imaginary part (corresponding to optical bandgap energy) is $3.1 \mathrm{eV}$. Practical calculations are carried out using SALMON, an open source software developed in our group $[3,4]$.

\section{Results and discussion}

Our calculated results of the electronic excitation energy, $\Delta E_{\mathrm{ex}}$, at a time after the field ends as a function of the laser intensity $I$ for several different frequencies $\omega$ are summarized in Fig.1(a), where the pulse duration is set to $T=15 \mathrm{fs}$ ( $5.5 \mathrm{fs}$ of FWHM) and $I$ is defined by $I=c \varepsilon_{0} E_{\max }^{2} / 2$ ( $E_{\max }$ is the maximum amplitude of the pulsed electric field).

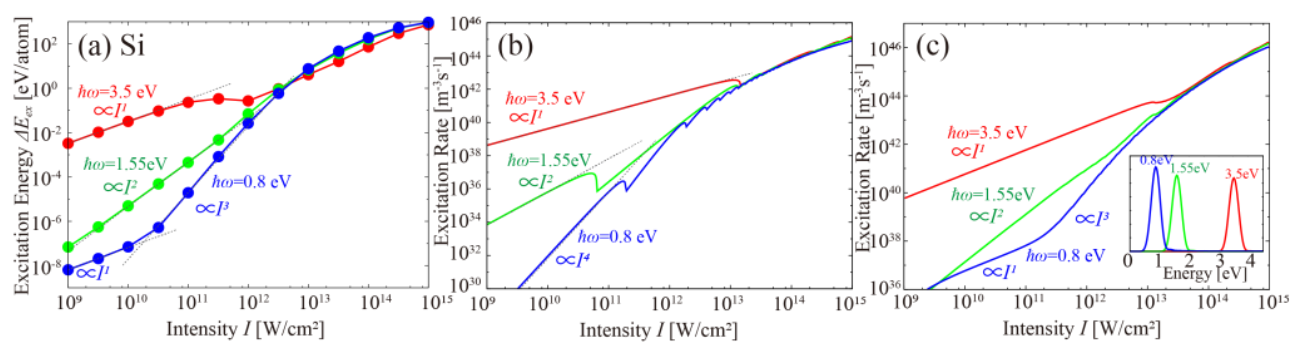

Fig. 1. (a) Electronic excitation energies in TDDFT calculation as a function of the intensity of the applied laser pulse with several frequencies. (b) Excitation rates by the Keldysh theory for continuous wave and (c) convoluted using power spectra of pulsed field as weight functions (the inset). The used parameters: $3.09 \mathrm{eV}$ of bandgap energy and $1.06 / 0.59$ a.u. of effective electron/hole masses, respectively.

The figure shows well-known power-law perturbation trend of multi-photon excitation $I^{N}$ depending on frequency (or photon energy) in lower intensity region. It is reasonable according to bandgap energy (around $3.1 \mathrm{eV}$ ), that is, the integer number $N$ coincides with the number of photons required to exceed the bandgap energy. There are however exceptions in the low frequencies data. At $\hbar \omega=0.8 \mathrm{eV}$, four photons are required to across the bandgap, while the excitation energy shows $N=3$ behavior. At very low intensity region, it shows $N=1$ behavior. These are discussed later. Increasing the intensity, the curves of the different frequencies intersect at a certain high intensity point. The excitation energies do not depend on the frequencies so much above the intersection point.

It is known that the Keldysh theory is capable of describing general features of ionization phenomena induced by strong, continuum fields, fully including nonlinear effects. To see quantitative comparison of our TDDFT results with the Keldysh theory, we show excitation rates in the Keldysh theory in Fig.1(b). It shows that the Keldysh theory reproduces the overall trend of the TDDFT results in Fig.1(a) very well even though the theory assumes continuous wave, indicating that the Keldysh model captures fundamental essence of the nonlinear interaction. However, the theory does not reproduce the above-mentioned exceptions in the plot of $\hbar \omega=0.8 \mathrm{eV}$. The discrepancy can be understood by the different 
time profile of the applied fields. To take account of the finite duration effect, we averaged the rate in the Keldysh theory utilizing the frequency distribution of the pulsed field used in the TDDFT calculation (Fig.1(c)). As seen from Fig.1(c), the exceptions, that is, $N=3$ and $N=1$ behaviors in the plot of $\hbar \omega=0.8 \mathrm{eV}$ are now reproduced being consistent with the TDDFT calculation. We find that the $I^{3}$ and $I^{I}$ behaviors instead of $I^{4}$ are due to high energy components of the pulsed field used in our calculation. We also see that small humps due to the ponderomotive effect in Fig.1(b) disappear by the averaging.

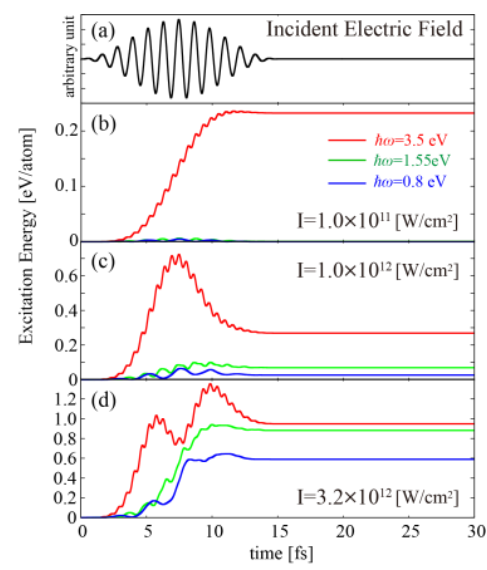

Fig. 2. Temporal development of $\Delta E_{\text {ex }}(t)$ during and after the pulse irradiation.

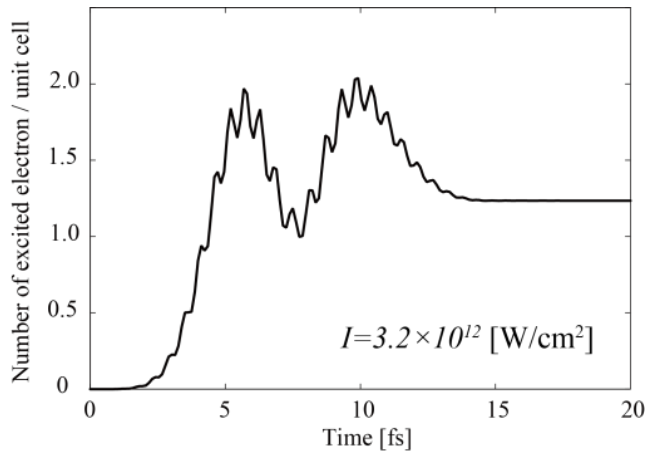

Fig. 3. The number of excited electrons for the data of $\hbar \omega=3.5 \mathrm{eV}$ and $I=3.2 \times 10^{12} \mathrm{~W} / \mathrm{cm}^{2}$.

Looking into details of Figs 1(a) and 1(c), another different feature is found in the plots of $\hbar \omega=3.5 \mathrm{eV}$. The excitation energy curve of the TDDFT calculation in Fig.1(a) shows somewhat a planar region just below the intersection point, around $10^{11}-10^{12} \mathrm{~W} / \mathrm{cm}^{2}$, while such behavior does not show up in the Keldysh theory in Fig.1(c). To figure out the reason of the difference, the time evolution of $\Delta E_{e x}(t)$ is shown in Fig.2. There appears an intriguing oscillation around the intensity of the intersection point (Figs.2(c) and (d)). The number of excited electrons shown in Fig.3 indicates the oscillation in Fig.2(d) is coming from substantial occupation change by resonant excitations, that is, the Rabi oscillation under a strong field. We consider the appearance of the planar region in the TDDFT calculation is related to the nonlinearity caused by the occupation change. The electronic excitation saturates during the irradiation and the energy transfer from the pulsed electric field to electrons becomes smaller than a simple estimate from the one-photon absorption.

This research was supported by JST-CREST under grant number JP-MJCR16N5, and by MEXT as a priority issue theme 7 to be tackled by using Post-K Computer, and by JSPS KAKENHI Grant Numbers 15H03674. Calculations are carried out at Oakforest-PACS at JCAHPC under the support by Multidisciplinary Cooperative Research Pro-gram in CCS, University of Tsukuba.

\section{References}

1. L. V. Keldysh, Soviet Phys. JETP, 20(5):1307 (1965).

2. G.F. Bertsch, J.-I. Iwata, A. Rubio, and K. Yabana, Phys. Rev. B62, 7998 (2000).

3. Web site of SALMON, http://salmon-tddft.jp/

4. M. Noda et al, arXiv:1804.01404. 